\title{
ON THE DEFINITION OF GRAPH INDEX
}

\author{
A. STOIMENOW \\ (Received 28 September 2011; accepted 20 November 2012; first published online 10 June 2013) \\ Communicated by C. Hodgson
}

\begin{abstract}
This paper discusses a flaw in Murasugi-Przytycki's Memoir 'An index of a graph with applications to knot theory' [Mem. Amer. Math. Soc. 106 (1993)]. We point out and partly fix a gap occurring in the proof of Murasugi-Przytycki's braid index inequalities involving the graph index. We explain why their notion of index fails to precisely reflect the reduction of Seifert circles by their diagram move, and redefine the index to account for that discrepancy.
\end{abstract}

2010 Mathematics subject classification: primary 57M25; secondary 05C10.

Keywords and phrases: skein polynomial, graph index, braid index, Bennequin surface.

\section{Introduction}

As an important part of the literature devoted to studying the braid index in the aftermath of the discovery of the Jones polynomial and its successors, MurasugiPrzytycki's Memoir [MP] introduces the notion of the index of a graph.

Their motivation stems from the relation discovered [Ya] between the braid index and the number of Seifert circles of a link diagram. They introduce a link diagram move to reduce the number of Seifert circles (see [MP, Figure 8.2], Figure 1, and Definition 2.1 below).

The Murasugi-Przytycki move reduces this number by one, and can (often) be applied repeatedly. However, the choice of move(s) is in general highly ambiguous, and the number of applicable moves depends heavily on this choice. The highest economy on Seifert circles is thus achieved when the number of moves is maximized.

Murasugi-Przytycki's definition of a graph index is based on a transformation of graphs, which models their diagram move on the level of Seifert graphs (see the proof of [MP, Lemma 8.6]). The precise definition of the index (and where a problem occurs with it) will be discussed in detail below, but it is important to notice already here that it entails a maximization over possible ways to modify the graph.

This work was partly funded by a grant of NRF (Korea).

(c) 2013 Australian Mathematical Publishing Association Inc. 1446-7887/2013 \$16.00 
Murasugi-Przytycki aim to obtain the inequality

$$
b(L) \leq s(D)-\operatorname{ind}(D) .
$$

Here $D$ is a diagram of $s(D)$ Seifert circles of a link $L$ with braid index $b(L)$, and ind $(D)$ is the index of the Seifert graph of $D$. The inequality (1.1) is one of the central results of [MP], and many applications there and elsewhere, for example [Oh], rely on it.

During our study of Murasugi-Przytycki's proof, we found a gap. It occurred when we wanted to understand the diagram move of [MP, Figure 8.2]. Murasugi-Przytycki seem to assume that Figure 8.2 is the general case, but we will explain that it is not. Taking care of the missing cases leads to a modified definition of index, which we call ind $\mathrm{i}_{0}$. Roughly speaking, the correction needed is that in certain situations some edges in the star of a vertex are not contracted (unlike in their procedure; see beneath Definition 3.2). Therefore, Murasugi-Przytycki's diagram move just proves instead of (1.1) that

$$
b(L) \leq s(D)-\operatorname{ind}_{0}(D) .
$$

Then the question naturally arises of how ind $(D)$ and $\operatorname{ind}_{0}(D)$ relate to each other. We will argue that

$$
\operatorname{ind}(D) \leq \operatorname{ind}_{0}(D),
$$

which justifies (1.1). This is necessary in order to rehabilitate the applications of this inequality inside and outside Murasugi-Przytycki's Memoir. We then speculated, based on our computational evidence, whether in fact

$$
\operatorname{ind}(D)=\operatorname{ind}_{0}(D)
$$

is always true. Later Traczyk [Tr] provided an argument that this is indeed true, by proving the reverse inequality to (1.3). Our understanding is that Traczyk's work thus clarifies an important point in the matter, but that this is not exactly what is needed (and it is not enough) to fix the error.

To do that, the explanation of ind $_{0}$ and (1.3) remains necessary, and succeeds only at a (minor) cost. This drawback is that Murasugi-Przytycki's definition of index loses its geometric meaning per se. It simplifies the true transformation of the Seifert graph under their diagram move, in a way which is a priori incorrect but (fortunately) $a$ posteriori turns out to still give the right quantity. This fact must be taken care of in subsequent applications of Murasugi-Przytycki's method, for example, in [MT].

The following account tries to explain the details.

\section{Braid representations and braid index}

The braid group $B_{n}$ on $n$ strands (or strings) is considered to be generated by the Artin standard generators $\sigma_{i}$ for $i=1, \ldots, n-1$. These are subject to relations of the type $\left[\sigma_{i}, \sigma_{j}\right]=1$ for $|i-j|>1$, which we call commutativity relations (the bracket denotes the commutator) and $\sigma_{i+1} \sigma_{i} \sigma_{i+1}=\sigma_{i} \sigma_{i+1} \sigma_{i}$, which we call Yang-Baxter (or YB) relations. 
A classical theorem of Alexander [Al] asserts that each link $L$ can be represented as $L=\hat{\beta}$, the closure of some braid $\beta$. We call $\beta$ a braid representation of $L$. The braid index $b(L)$ of a link $L$ is the smallest number of strands among all braid representations of $L$ (see [Mo, FW, Mu]). A braid $\beta$ on $b(L)$ strands with $\hat{\beta}=L$ is called a minimal braid (representation) of $L$.

The skein polynomial $P[\mathrm{~F}, \mathrm{LM}]$ is a Laurent polynomial in two variables $l$ and $m$ of oriented knots and links and can be defined by being 1 on the unknot and by the (skein) relation

$$
l^{-1} P(\nearrow)+l P(\searrow)=-m P(\nearrow \circlearrowright)
$$

As usual, the three fragments depict link diagrams identical elsewhere. The convention uses the variables of $[\mathrm{LM}]$, but differs from the one adopted by the interchange of $l$ and $l^{-1}$.

Let $P \in \mathbb{Z}\left[l^{ \pm 1}, m^{ \pm 1}\right]$. The minimal respectively maximal $l$-degree $\min \operatorname{deg}_{l} P$ respectively $\max _{\operatorname{deg}_{l}} P$ is the minimal respectively maximal exponent of $l$ in a monomial (with nonzero coefficient) in $P$. Let $\operatorname{span}_{l} P=\max \operatorname{deg}_{l} P-\min \operatorname{deg}_{l} P$.

A crossing as on the left in (2.1) has writhe (or skein sign) 1 and is called positive. A crossing as in the middle of (2.1) has writhe -1 and is called negative. The writhe $w(D)$ of a link diagram $D$ is the sum of writhes of all its crossings.

The replacement of a (positive or negative) crossing by the the rightmost expression in (2.1) is called smoothing out. When all crossings of $D$ are smoothed out, we have a collection of loops called Seifert circles. When a crossing in $D$ is reinstalled by undoing the smoothing operation, it can be regarded as connecting two (distinct) Seifert circles. Let $s(D)$ be the number of Seifert circles of $D$.

In $[\mathrm{Mo}, \mathrm{FW}]$ it was proved for the skein polynomial $P(l, m)$ that

$$
\frac{1}{2} \operatorname{span}_{l} P(L)+1 \leq b(L)
$$

the Morton-Williams-Franks (MWF) inequality.

It was soon noticed that for many links the MWF inequality is sharp (that is, an equality), and for a while it was conjectured that this would be so for all alternating links. Murasugi-Przytycki disproved this conjecture, obtaining (among others) an 18 crossing counterexample alternating knot.

The inequality (2.2) results from two other inequalities, due to Morton, namely that for a diagram $D$, we have

$$
1-s(D)+w(D) \leq \min \operatorname{deg}_{l} P(D) \leq \max _{\operatorname{deg}_{l}} P(D) \leq s(D)-1+w(D) .
$$

Williams-Franks showed these inequalities for the case of braid representations. Later it was observed from the algorithms of Yamada [Ya] and Vogel [Vo] that the braid version is actually equivalent to, and not just a special case of, the diagram version. These algorithms allow any diagram $D$ to be turned into a braid diagram without altering $s(D)$ and $w(D)$. Nonetheless we will refer to (2.3) as 'Morton's inequalities'. 
With the relation to Seifert circles at hand, Murasugi-Przytycki's effort centers around minimizing by isotopy their number for a given link diagram. For this purpose they introduce a move as follows.

Definition 2.1 (See [MP, Figure 8.2] and Figure 1). Let $D$ be a diagram of a link $L$ and $e$ a crossing which connects as a unique crossing two Seifert circles $v$ and $w$. Choose one of these two Seifert circles, say, v. The Murasugi-Przytycki move takes the undercrossing strand at $e$ and reroutes it to a long arc of undercrossings going along some Seifert circles neighbored (that is, connected by crossings) to $v$ in $D$. This way one obtains another diagram $D^{\prime}$ of $L$ with $s\left(D^{\prime}\right)=s(D)-1$.

The resulting long arc in $D^{\prime}$ is indicated by a dashed line in Figure 1. It is supposed to pass below any other strand it crosses. Note that one can also reroute the overpass at $e$, obtaining a (generally) different diagram $D^{\prime \prime}$ of $L$ with one Seifert circle less than $D$. However, the graph theory discussed below will imply that, for the purpose of minimizing the number of Seifert circles, the two new diagrams $D^{\prime}$ and $D^{\prime \prime}$ do equally well.

This move aims at an improvement of the inequalities (2.3) (restated below in Proposition 3.3), which allows one to settle the braid index problem for many links (see Theorem 7.1, Conjecture 3.5, and also [Oh]). For the purpose of simplifying the work with their move using Seifert graphs, Murasugi-Przytycki developed the concept of index of a graph. We recall some main points of their work, referring to [MP] for further details.

\section{Graph index}

Graphs will be finite. It will be no restriction to assume that they are planar, that is, to admit a planar embedding. (We later remark on the ambiguity of the planar embedding.) We allow different edges to connect the same two vertices. Such edges will be counted (and in certain cases, treated) separately. We thus understand a multiple edge as a set consisting of the edges connecting the same two vertices.

An edge is simple if no other edge connects the same two vertices. Such an edge will be sometimes denoted by its two vertices (order irrelevant).

Loop edges can also be allowed, but are not very relevant.

Definition 3.1. Let $G$ be a connected graph. For a vertex $v$ in $G$ let the star star $v$ of $v$ be the set of edges in $G$ incident from $v$, that is, those for which $v$ is one of the endpoints. Let $G_{v}=G / v$ be the graph obtained from $G$ by contracting star .

Let $G \backslash v$ be the graph obtained from $G$ by deleting star $v$, and additionally $v$ itself. (When we delete an edge, we understand that any vertex it is incident to is not to be deleted along the way.) We call $v$ a cut vertex of $G$, if $G \backslash v$ is disconnected.

Let $G$ be a connected signed graph; 'signed' will mean for us that each edge carries a sign + or - . There is no problem in extending the various introduced graph operations to signed graphs. 
Definition 3.2. We define (recursively) a sequence of edges $\mu=\left(e_{1}, \ldots, e_{n}\right)$ to be independent in a graph $G$ if the following conditions are satisfied.

1. The empty (edge) sequence is independent per definition.

2. Let $e_{1}$ connect vertices $v_{1}$ and $v_{2}$. Then we demand that $e_{1}$ is simple, that is, there is no other edge connecting $v_{1,2}$, and that $e_{2}, \ldots, e_{n}$ is independent in (one of) $G_{v_{1}}$ or $G_{v_{2}}$ (in particular, $\left\{e_{2}, \ldots, e_{n}\right\}$ is disjoint from at least one of star $v_{1}$ and $\operatorname{star} v_{2}$, respectively).

An independent set is a set of edges admitting an ordering as an independent sequence.

The index ind $(G)$, the positive index $\operatorname{ind}_{+}(G)$, respectively negative index ind $(G)$ of $G$, are defined as the maximal length of an independent edge set (or sequence), independent positive, and independent negative edge set/sequence in $G$, respectively. A sequence is maximal independent if it realizes the index of $G$.

Now to each link diagram $D$ we associate its Seifert graph $G=\Gamma(D)$, which is a planar bipartite signed graph. It consists of a vertex for each Seifert circle in $D$ and an edge for each crossing, connecting two Seifert circles. Each edge is signed by the writhe (or skein sign) of the crossing it represents, as explained underneath Equation (2.1). Note that several edges between the same vertices can thus occur, and they may also carry different signs. We will for convenience sometimes identify crossings/Seifert circles of $D$ with edges/vertices of $G$.

Then we can set $\operatorname{ind}_{( \pm)}(D)=\operatorname{ind}_{( \pm)}(\Gamma(D))$. Murasugi-Przytycki claim the following proposition.

Proposition 3.3 (See [MP, (8.4) and (8.8)]). If $D$ is a diagram of an oriented link L, then

$$
\begin{aligned}
\max _{\operatorname{deg}_{l} P(L)} & \leq w(D)+s(D)-1-2 i n d_{+}(D) \\
\min \operatorname{deg}_{l} P(L) & \geq w(D)-s(D)+1+2 i n d_{-}(D) \\
b(L) & \leq m p b(D):=s(D)-\operatorname{ind}(D) .
\end{aligned}
$$

An important operation on diagrams studied in relation to the index is this of Murasugi sum decomposition (see [Cr, Section 1]). On the level of Seifert graphs it corresponds (mainly) to block decomposition.

Definition 3.4. The join (or block sum) $G_{1} * G_{2}$ of two graphs $G_{1}$ and $G_{2}$ is defined by

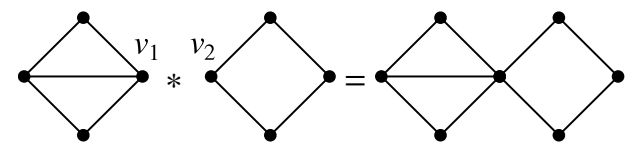

This operation depends on the choice of a vertex $v_{i}$ in each one of the graphs $G_{i}$. (Although this dependence will not be notationally highlighted, it should be kept in mind.)

Every connected nontrivial (that is, with at least one edge) graph $G$ can be written as a join $G_{1} * \cdots * G_{n}$ for some nontrivial connected graphs $G_{i}$, such that no $G_{i}$ has a cut vertex. We call $G_{i}$ the block components or join factors of the graph $G$. 
The precise relation between block and Murasugi sum decomposition is as follows. A Seifert circle in D is separating if it has crossings attached to it from both its interior and exterior. A diagram with no separating Seifert circles is called special. The blocks of $D$ are the connected sum components of the pieces of $D$ obtained by Murasugi sum decomposition of $D$ along its separating Seifert circles. See for this [Cr, Section 1], but keep in mind that Cromwell's definition of blocks does not take into account connected sum decomposition. (Thus some of Cromwell's blocks can decompose into several blocks in our sense.)

Then each block component of $\Gamma(D)$ is the Seifert graph of a block of $D$. (It is for the sake of this analogy that we alter here Cromwell's definition.)

If $G=\Gamma(D)$ is a block component itself (that is, has no cut vertex, and $D$ is prime and special), one can recover $D$ uniquely from a concrete planar embedding of $G$ (both regarded up to moves in $S^{2}$ ). It is helpful, for example, in comparing Figures 1 and 2 below, to keep in mind the correspondence between a block of $D$ and the planar embedding of its Seifert graph. However, under block sum, there is little sense in dwelling upon planar embeddings. This is why the block sum (3.4) of two graphs $G_{1}$ and $G_{2}$ is understood to depend on not more than the choice of vertices $v_{i}$ in $G_{i}$.

For any diagram $D$, we have

$$
\operatorname{ind}_{+}(D)+\text { ind }_{-}(D) \geq \operatorname{ind}(D) .
$$

For alternating (and more generally homogeneous $[\mathrm{Cr}]$ ) diagrams $D$ equality holds, because each join factor of $\Gamma(D)$ contains only edges of the same sign. This implies that if in such diagrams (3.1), (3.2) are sharp, then (2.2) and (3.3) become sharp, too.

Conjecture 3.5 (Murasugi-Przytycki). If D is an alternating diagram of a link $L$, then $b(L)=\operatorname{mpb}(D)$.

\section{Hidden Seifert circle problem}

Now we must understand the move of Murasugi-Przytycki that corresponds to the choice of a simple edge $e$ and the contraction of the star of $v$ in $G$. To set the record straight, we should mention that this move was also considered, apparently simultaneously and independently, by Chalcraft [Ch], although only as a secondary issue. With this understanding, we will still refer to it below as the Murasugi-Przytycki move.

This move is shown in [MP, Figure 8.2], but see Figure 1 below for a more authentic situation. Let $D$ be the diagram before the move and $D^{\prime}$ the diagram resulting from it. Let us for simplicity identify an edge with its crossing and a vertex with its Seifert circle (see the remark above Proposition 3.3). In this language, the move of MurasugiPrzytycki eliminates one crossing, corresponding to $e$. The crossings of the other edges $e^{\prime} \neq e$ incident to $v$ do not disappear under the Murasugi-Przytycki move. Instead, they become in $D^{\prime}$ parts of join factors of $\Gamma\left(D^{\prime}\right)$ that correspond to a Murasugi summand on the opposite side of the modified Seifert circle. See the proof of [MP, Lemma 8.6], and Figures 1 and 2 below. 


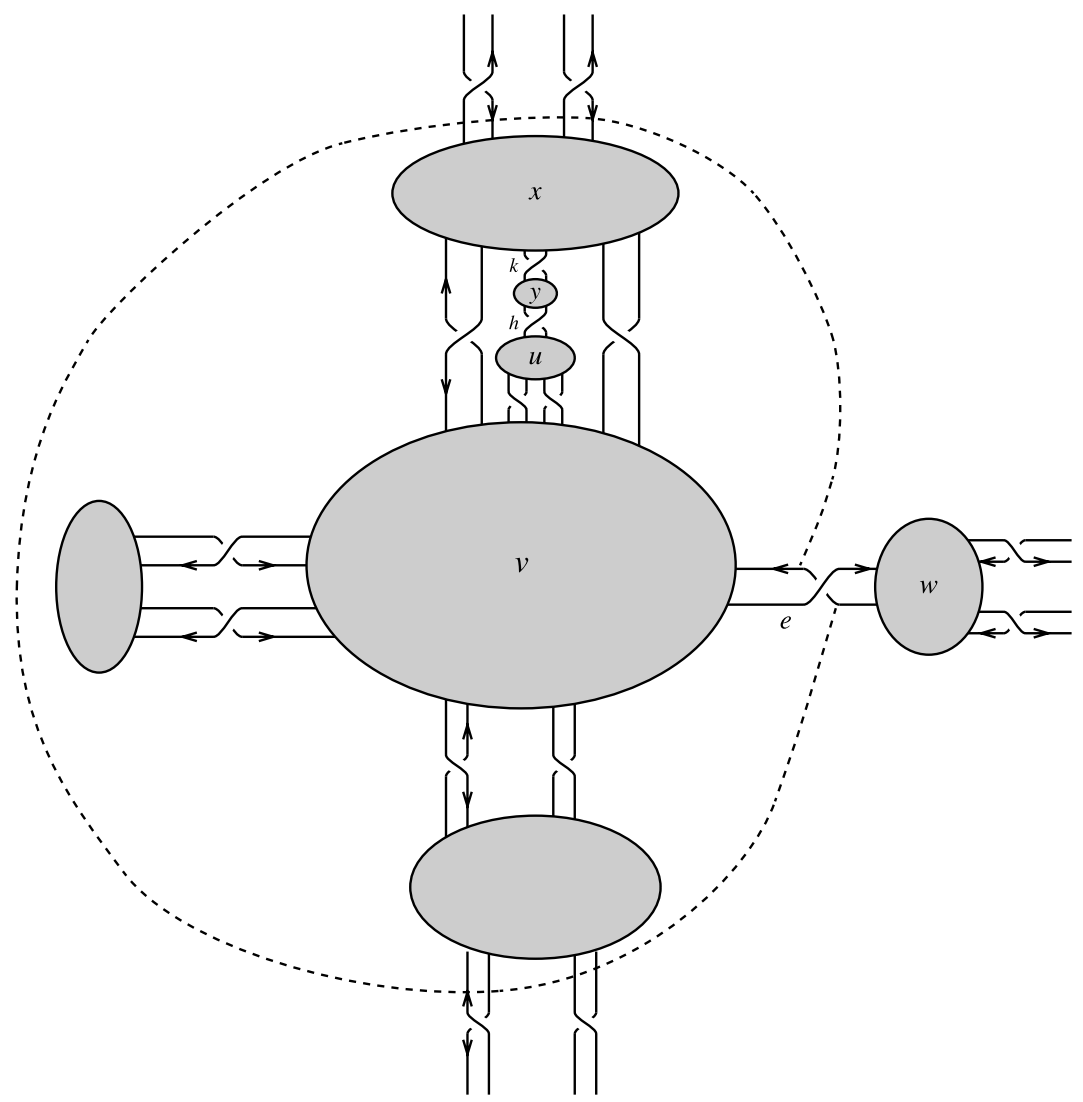

FIgure 1. A move of Murasugi-Przytycki, where the relayed strand (dotted line) does not go along a Seifert circle (denoted as $u$ ) adjacent to $v$. The Seifert circles are depicted in gray to indicate that their interiors may not be empty.

The subtlety, which seems to have been overlooked in the proof of [MP], is illustrated in Figures 1 and 2. The Seifert circles adjacent to $v$ may be nested in $D$ in such a way that relaying the arc of $v$ by the move, one does (and can) not go along all Seifert circles adjacent to $v$. In the Seifert graph $G^{\prime}=\Gamma\left(D^{\prime}\right)$ of $D^{\prime}$ some of the edges incident to $v$ in $G=\Gamma(D)$ may not enter, as written in the proof of [MP, Lemma 8.6], into block components that are 2-vertex graphs (with a multiple edge).

Still we see that contracting the star of $v$ in $G=\Gamma(D)$, we obtain a graph $\tilde{G}=G / v$, which is a contraction of $G^{\prime}=\Gamma\left(D^{\prime}\right)$. (We will later describe exactly how $G^{\prime}$ is constructed from $G$, but let us for the time being use the easier to obtain $G / v$ instead.) Here contraction $\tilde{G}$ of a graph $G^{\prime}$ means that $\tilde{G}$ is obtained from $G^{\prime}$ by contracting some (possibly several or no) edges, and we allow multiple edges in $G^{\prime}$ to be contracted (by doing so simultaneously with all edges they consist of).

More precisely, the difference between the block component of $\tilde{G}$ and $G^{\prime}$ is that in the last block component of $G^{\prime}$ in Figure 2 the star of $v$ is contracted to obtain the block 


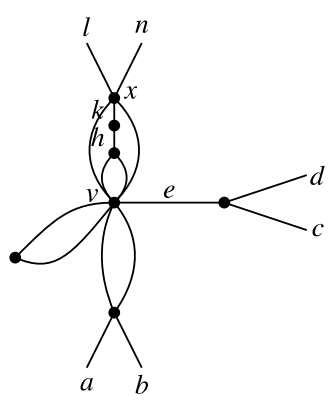

$G=\Gamma(D)$

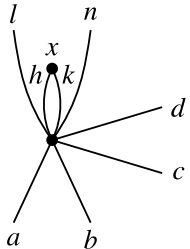

$\tilde{G}=G / v$
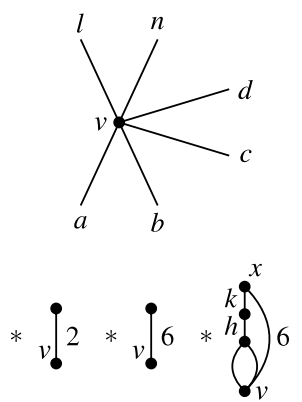

$G^{\prime}=\Gamma\left(D^{\prime}\right)$

FIGURE 2. The various Seifert graphs of the diagrams related to the move of Murasugi-Przytycki in Figure 1, in the case when the relayed strand does not go along all Seifert circles adjacent to $v$. The graph of $D^{\prime}$ is given in its block decomposition, which corresponds to the Murasugi sum decomposition along the newly created Seifert circle. For simplicity, we display a multiple edge by attaching the multiplicity to the edge drawn as simple (otherwise, a letter attached just indicates the name).

component of $\tilde{G}$ consisting of edges $h$ and $k$. So for the proof of [MP, Lemma 8.6] and (3.3), we actually need the following lemma.

LemMa 4.1. If a graph $H^{\prime}$ is a contraction of $H$, then ind $\left(H^{\prime}\right) \leq \operatorname{ind}(H)$.

Proof. We prove that each independent set of edges in $H^{\prime}$ is independent in $H$. We do this inductively over the number of vertices of $H$.

Let $e_{1}, \ldots, e_{n}$ be independent in $H^{\prime}$. Then $e_{2}, \ldots, e_{n}$ are independent in $H^{\prime} / v_{1}$, for some vertex $v_{1}$ to which $e_{1}$ is incident. All edges $e_{1}, \ldots, e_{n}$ exist in $H$, and so does $v_{1}$. (During the contractions that turn $H$ into $H^{\prime}$, the vertex $v_{1}$ in $H$ may be identified with others.)

Now $H^{\prime} / v_{1}$ is a contraction of $H / v_{1}$, and thus by induction assumption, $e_{2}, \ldots, e_{n}$ are independent in $H / v_{1}$. Moreover, since $e_{1}$ is simple in $H^{\prime}$, it is simple in $H$. (Contractions cannot eliminate multiple edges except by contracting them.) Thus, $e_{1}, \ldots, e_{n}$ are independent in $H$, as we wanted.

With the lemma, we will establish in Lemma 5.4 below that we can reduce at least $\operatorname{ind}(D)$ Seifert circles by Murasugi-Przytycki moves, and (3.3) is recovered.

Still it should be understood that the contraction of a vertex fails to model in full accuracy the Murasugi-Przytycki diagram move.

\section{Modifying the index}

It becomes necessary to understand exactly the transformation of the Seifert graph $G$ under the move of Murasugi-Przytycki. We describe it now, also filling in the detail overlooked by them.

Now we use marked graphs. This means that edges have a $\mathbb{Z}_{2}$-graduation. Each edge either carries a mark (and is marked) or not (and is unmarked). Its marking is the 
status according to this graduation. This distinction is different from the $+/-$ signing. However, marked edges carry no sign, so that when signs are relevant, one should distinguish edges into positive, negative (unmarked) and marked edges.

We assume for the rest of the exposition that $G$ is bipartite. Thus $G$ has no loop edges (isthmusses) and no cycles of length 3, which avoids some technical difficulties.

In the initial (Seifert) graph all edges are unmarked (and carry, if relevant, the sign of their corresponding crossing). A marked edge is to be understood as one that cannot be chosen as an edge $e$. It corresponds to a multiple edge.

Definition 5.1. We choose a nonmarked edge $e$ and a vertex $v$ of $e$. Let $w$ be the other vertex of $e$ (see Figure 1). We define the notion on the opposite side to $e$ as follows.

A vertex $y \neq v, w$ is on the opposite side to $e$ if there is a vertex $x \neq v, w, y$ adjacent to $v$ such that $y$ and $w$ are in different connected components of $(G \backslash v) \backslash x$.

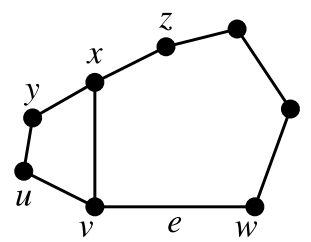

(Here 'I' stands for the deletion of a vertex together with all its incident edges - but not its adjacent vertices; see Definition 3.1.) Otherwise we say $y$ is on the same side ae.

The meaning of this distinction is that the Murasugi-Przytycki move lays the arc along a Seifert circle $x$ adjacent to (the Seifert circle of) $v$, if $x$ is on the same side as $e$. This move affects the crossings that connect $x$ to $v$, or to a Seifert circle $z$ on the same side as $e$.

Definition 5.2. Let $G$ be a marked graph, $v$ a vertex of $G$, and $e$ a simple unmarked edge between $v$ and another vertex $w$. We define now the marked graph $G / e^{v}$.

The vertices of $G / e^{v}$ are those of $G$ except $w$. The edges and their markings are chosen by copying those in $G$ as follows. Let an edge $e^{\prime}$ in $G$ connect vertices $v_{1,2}$.

Case 1. $v$ is among $v_{1,2}$, say $v=v_{1}$.

Case 1.1. If the other vertex $v_{2}$ of $e^{\prime}$ is $w$ (that is $e=e^{\prime}$ ), then $e^{\prime}$ is deleted.

Case 1.2. If $v_{2}$ is on the opposite side to $e$, then $e^{\prime}$ is retained in $G / e_{e} v$ with the same marking.

Case 1.3. If $v_{2}$ is on the same side as $e$, then $e^{\prime}$ is retained in $G / e^{v}$, but marked.

Case 2. $v$ is not among $v_{1,2}$.

Case 2.1. If none of $v_{1,2}$ is adjacent to $v$, then $e^{\prime}$ retains in $G / e^{v}$ the same vertices and marking.

Case 2.2. One of $v_{1,2}$, say $v_{1}$, is adjacent to $v$. (Then $v_{2}$ is not adjacent to $v$ by bipartacy.) 
Case 2.2.1. If $v_{1}=w$, then change $v_{1}$ to $v$ in $G / e^{v}$, and retain the marking.

Case 2.2.2. So assume next $v_{1} \neq w$. If $v_{2}$ is on the opposite side to $e$, then retain $v_{1,2}$ and the marking.

Case 2.2.3. If $v_{2}$ is on the same side as $e$, then we change $v_{1}$ to $v$, and retain the marking. (Note that by bipartacy, if $v_{2}$ is on the same side as $e$, then so must be $v_{1}$.)

In the case of a signed (unmarked) edge $e^{\prime}$, the sign is copied (even if vertices are changed), except in the case 1.3 , when $e^{\prime}$ receives a mark. In this case the sign of $e^{\prime}$ is deleted.

Since a mark will indicate for us only that the edge cannot be chosen as $e$, the resulting graph $G / e_{e} v$ may be reduced by turning a multiple edge into a simple marked one. (This also makes it irrelevant to create a multiple edge in case 1.3.)

Definition 5.3. We can extend the Definition 3.2 of $\operatorname{ind}(G)$ to marked graphs $G$ by requiring in point 2 that $e$ be simple and unmarked, and allowing at every stage the option that a multiple edge can be turned into a simple marked one. Then $\operatorname{ind}(G)$ coincides with the one previously defined if all edges of $G$ are unmarked (in particular $\operatorname{ind}(D)=\operatorname{ind}(\Gamma(D))$ when all edges of $\Gamma(D)$ are understood unmarked).

If we further replace in Definition 3.2 the two occurrences of $G_{v_{i}}=G / v_{i}$ by $G / e_{e} v_{i}$, as given in Definition 5.2, then we define the corresponding notions of 0-independent edges and the modified index $\operatorname{ind}_{0}(G)$. Again we set $\operatorname{ind}_{0}(D)=\operatorname{ind}_{0}(\Gamma(D))$ with all edges of $\Gamma(D)$ unmarked.

If one requires that in a set of 0 -independent edges all be positive or negative, one obtains the modifications ind $_{0, \pm}$ of ind $_{ \pm}$.

With this definition, we obtain (1.2). The property (1.3) can be proved by induction.

Lemma 5.4. For every marked graph $G$ we have ind $(G) \leq \operatorname{ind}_{0}(G)$.

Proof. We prove inductively over the vertex number of $G$ that each independent set of edges in $G$ is also 0 -independent.

Let $e_{1}, \ldots, e_{n}$ be independent in $G$. Thus $e_{1}$ is simple and unmarked in $G$, and $e_{2}, \ldots, e_{n}$ are independent in $G / v_{1}$. Since $G / v_{1}$ is a contraction of $G / e^{v_{1}}$, by the proof of Lemma 4.1, the edges $e_{2}, \ldots, e_{n}$ are independent in $G / e_{e} v_{1}$. By induction assumption they are thus 0 -independent in $G / e_{e} v_{1}$. Therefore, $e_{1}, \ldots, e_{n}$ are 0 -independent in $G$.

This fixes Murasugi-Przytycki's proof of [MP, Lemma 8.6]. A similar fix works for (3.1) and (3.2).

We speculated whether in fact (1.2) can be stronger than (1.1). If (1.4) is false, then conjecture 3.5 is also false. We explained, though, that indeed (1.4) is true (and was proved by Traczyk as a follow-up to our note). Still our (much more awkward) definition of the modified index is needed, at least for the scope of this paper, in order to prove (1.3) or (1.4), and fix the gap in [MP]. 
Also, if one likes to keep the correspondence between (Seifert) graph and diagram, one must accept the circumstance that (in general) not all of starv is to be contracted. The idea of using vertex contraction (straightforwardly, following [MP]) appeared in at least one further paper, [MT]. To prevent problems from propagating, we feel some priority is justified to our point of caution. This was an additional motivation for the present correction.

\section{Simple properties of the modified index}

The important difference of ind $_{0}$ to ind lies in not affecting edges in case 1.2. The treatment of vertices on the opposite side to $e$, the technical detail missed by Murasugi-Przytycki, does not affect the result significantly, yet it creates a great deal of calculation overhead (which we experienced in attempts to use the possibly better estimate (1.2) prior to Traczyk's proof of (1.4)). Note, however, that it implies the additivity of ind $_{0}$ under block sum in an easier (and much more natural) way than Murasugi-Przytycki's corresponding statement for ind.

Definition 6.1. A marked graph is not 2-connected if it has an unmarked edge whose deletion disconnects it. If $G$ is not 2-connected, there is a plane curve intersecting $G$ in a single, and unmarked, edge. We call such a curve a separating curve.

Note that the initial (unmarked Seifert) graph of $D$ is 2-connected because we can assume $D$ has no nugatory crossings.

LeMma 6.2. If $G$ is 2-connected, so is $G / e^{v}$.

Proof. We assume to the contrary that $G / e^{v}$ is not 2-connected. Let $e^{\prime}$ be a disconnecting edge. So there is a separating curve $\gamma$ that intersects $G / e_{e} v$ only in $e^{\prime}$. The only edges in $G / e_{e} v$ which do not exist in $G$ are of the type $v z$ in (5.1). That is, $z$ is a vertex on the same side as $e$, adjacent to a vertex $x$ adjacent to $v$ in $G$. (Note that when $x=w$, then all $z$ adjacent to $w$ are on the same side as $e$.) By Definition 5.1, the property of $z$ being on the same side as $e$ implies that there is a cycle in $G$ containing the edges $e=v w, v x$ and $x z$. In $G / e_{e} v$, this cycle is shortened when $x z$ is replaced by $v z$ (and $e$ contracted). Thus $v z$ belongs to a cycle in $G / e_{e} v$, and cannot disconnect $G / e_{e} v$.

Therefore, $e^{\prime}$ persists in $G$. It must be unmarked in $G$, since the move from $G$ to $G / e^{v}$ never deletes marks. Thus the curve $\gamma$ must intersect $G$ in some other edge. The only edges added in $G$ when recovering it from $G / e^{v}$ (except that $e$ is decontracted) are of the form $x z$ in (5.1) (with $x$ a vertex adjacent to $v$, and $z$ a vertex adjacent to $x$ on the same side as $e$ ). Then $\gamma$ passes in $G$ through a cycle as the right one in (5.1) (the one containing $z, x, v, w$ in consecutive order; note that $z \neq w$ by bipartacy). In $G / e_{e} v$ this cycle is changed only by replacing $x z$ and $v x$ by $v z$ (and contracting $e$ ). The only way $\gamma$ can avoid this cycle in $G / e v$ is that $\gamma$ passes through $e^{\prime}=v x$ in $G / e v$ (and $G$ ). But by construction $v x$ is marked in $G / e v$, and $\gamma$ is not a separating curve, a contradiction.

It is easy to see that $G_{1} * G_{2}$ is 2 -connected if and only if both $G_{1}$ and $G_{2}$ are. This is true regardless of how (that is, at which vertices) ' $*$ ' is performed. 
Lemma 6.3. If $G_{1,2}$ are 2-connected, then ind $d_{0}\left(G_{1} * G_{2}\right)=\operatorname{ind}_{0}\left(G_{1}\right)+\operatorname{ind}_{0}\left(G_{2}\right)$.

PROOF. It is enough to see that the contraction procedure of an edge $e$ in $G_{1}$ does not affect edges or markings in $G_{2}$, except possibly the change of vertex at which the block sum $G_{1} * G_{2}$ is performed.

Let $v, w$ be the ends of $e$, and we consider the building of $G / e_{e} v$ for $G=G_{1} * G_{2}$. Let $z$ be the (cut) vertex of $G$ at which the block sum $G_{1} * G_{2}$ is performed.

If $z \neq v$ is not adjacent to $v$, then nothing is changed in $G_{2}$ when building $G /{ }_{e} v$.

Next assume $z=v$. The vertex $v$ must be adjacent to at least one more vertex $x \neq w$ in $G_{1}$ (else $G_{1}$ is not 2-connected or $e$ is multiple). Then we see with this choice of $x$ in Definition 5.1 that the vertices in $G_{2}$ except $v$ lie on the opposite side to $e$. Thus building $G / e^{v}$ does not affect $G_{2}$.

Finally assume $z \neq v$, but $z$ is adjacent to $v$. If $z=w$ is the other end of $e$, then in $G / e v$ all edges incident in $G_{2}$ to $w$ are redirected to $v$ with the same marking, and so $G_{2}$ is not affected. If $z \neq w$, then choosing $z$ for $x$ in Definition 5.1, we see that all vertices of $G_{2}$ except $z$ are on the opposite side to $e$. Thus nothing of $G_{2}$ is affected by building $G / e^{\nu}$.

\section{Outline of applications}

We conclude by briefly outlining the context in which the above problem was encountered.

Beside the standard presentation of the braid groups using Artin's generators $\sigma_{i}$, another presentation has been studied for some time [BKL] by means of an extended set of band generators (and their inverses)

$$
\sigma_{i, j}^{ \pm 1}=\sigma_{i} \ldots \sigma_{j-2} \sigma_{j-1}^{ \pm 1} \sigma_{j-2}^{-1} \ldots \sigma_{i}^{-1}
$$

for $1 \leq i<j \leq n$. (Note that $\sigma_{i}=\sigma_{i, i+1}$.)

A representation of a braid $\beta$, and its closure link $L=\hat{\beta}$, as word in $\sigma_{i, j}^{ \pm 1}$ is called a band representation. A band representation of $\beta$ spans naturally a Seifert surface of the link $L$ : one glues disks into the strands, and connects them by half-twisted bands along the $\sigma_{i, j}$. The resulting surface is called the braided Seifert surface of $L$.

A minimal genus Seifert surface of $L$ occurring in the form of a braided Seifert surface is called a Bennequin surface. This term was coined by BirmanMenasco [BM] in honor of Bennequin, who had proved in [Be] that such surfaces exist for 3-braid links on a minimal (that is, 3-strand) braid. It is known that not all links (or knots) carry a Bennequin surface on a minimal braid.

Murasugi-Przytycki's work (and its present correction) will be applied to obtain the following result.

THEOREM 7.1. Any alternating knot of genus up to 4 or of at most 18 crossings:

(a) makes the Morton-Williams-Franks inequality (2.2) sharp (that is, an equality), except if it is the Murasugi-Przytycki knot or its mutant; 
(b) satisfies Conjecture 3.5 (for at least one alternating diagram D); and

(c) carries a Bennequin surface on a minimal braid.

The proof uses, among others, a computer implementation of Murasugi-Przytycki's graph algorithm. Details will be explained in a subsequent paper.

\section{Acknowledgements}

I would like to thank J. C. Cha and P. Traczyk for some helpful remarks.

\section{References}

[Al] J. W. Alexander, 'A lemma on systems of knotted curves', Proc. Natl. Acad. Sci. USA 9 (1923), 93-95.

[Be] D. Bennequin, 'Entrelacements et équations de Pfaff', Soc. Math. de France, Astérisque 107-108 (1983), 87-161.

[BKL] J. S. Birman, K. Ko and S. J. Lee, 'A new approach to the word and conjugacy problems in the braid groups', Adv. Math. 139(2) (1998), 322-353.

[BM] J. S. Birman and W. W. Menasco, 'Studying links via closed braids II: on a theorem of Bennequin', Topology Appl. 40(1) (1991), 71-82.

[Ch] D. A. Chalcraft, 'On the braid index of links with nested diagrams', Math. Proc. Cambridge Philos. Soc. 111(2) (1992), 273-281.

[Cr] P. R. Cromwell, 'Homogeneous links', J. Lond. Math. Soc. (2) 39 (1989), 535-552.

[FW] J. Franks and R. F. Williams, 'Braids and the Jones-Conway polynomial', Trans. Amer. Math. Soc. 303 (1987), 97-108.

[F] P. Freyd, J. Hoste, W. B. R. Lickorish, K. Millett, A. Ocneanu and D. Yetter, 'A new polynomial invariant of knots and links', Bull. Amer. Math. Soc. 12 (1985), 239-246.

[LM] W. B. R. Lickorish and K. C. Millett, 'A polynomial invariant for oriented links', Topology 26(1) (1987), 107-141.

[MT] J. Malesic and P. Traczyk, 'Seifert circles, braid index and the algebraic crossing number', Topology Appl. 153(2-3) (2005), 303-317.

[Mo] H. R. Morton, 'Seifert circles and knot polynomials', Proc. Camb. Phil. Soc. 99 (1986), 107-109.

[Mu] K. Murasugi, 'On the braid index of alternating links', Trans. Amer. Math. Soc. 326(1) (1991), 237-260.

[MP] K. Murasugi and J. Przytycki, 'An index of a graph with applications to knot theory', Mem. Amer. Math. Soc. 106 (1993).

[Oh] Y. Ohyama, 'On the minimal crossing number and the braid index of links', Canad. J. Math. 45(1) (1993), 117-131.

[Tr] P. Traczyk, 'Reducing braid index by Murasugi-Przytycki operation', J. Knot Theory Ramifications 20(1) (2011), 223-229.

[Vo] P. Vogel, 'Representation of links by braids: A new algorithm', Comment. Math. Helv. 65 (1990), 104-113.

[Ya] S. Yamada, 'The minimal number of Seifert circles equals the braid index', Invent. Math. 88 (1987), 347-356.

\section{A. STOIMENOW, Department of Mathematics, Keimyung University, Darseo-Gu, Dalgubeoldaero 2800, Daegu 704-701, Korea \\ e-mail: stoimeno@stoimenov.net}

